\title{
Utility of lumbar puncture after a normal brain CT scan in patients presenting to the emergency department with suspected Subarachnoid Haemorrhage, a new more rational approach?
}

Pouryahya P (1,2,4), Haydon R (3,4), Meyer A (1,2,4), Easaw-Mamutil N (4), Zhi Shawn Tan Y (4), Teng Hui Wen G (4).

1) Casey hospital, Emergency Department, Program of Emergency Medicine, Monash Health, Victoria

2) Monash Emergency Research Collaborative, School of Clinical Sciences, Monash Health, Monash University, Victoria

3) Monash Medical Centre, Emergency Department, Program of Emergency Medicine, Monash Health, Victoria

4) Faculty of Medicine, Nursing and Health Sciences, Monash University, Victoria

\author{
Corresponding author: \\ Dr. Pourya Pouryahya \\ Casey hospital, 62 Kangan drive, Berwick, Victoria 3806, Australia \\ email: Pourya.Pouryahya@monashhealth.org \\ Pourya.Pouryahya@monash.edu
}

\begin{abstract}
Background:

The diagnostic approach for patients presenting to Emergency Departments (EDs) with headaches suspicious for subarachnoid haemorrhage (SAH) remains challenging. Modern third generation computed tomography has been shown to be extremely sensitive in identifying SAH and may eliminate the need for lumbar puncture which is an invasive, time consuming procedure with limited accuracy and complications.
\end{abstract}

This is the author manuscript accepted for publication and has undergone full peer review but has not been through the copyediting, typesetting, pagination and proofreading process, which may lead to differences between this version and the Version of Record. Please cite this article as doi: 10.1111/1742-6723.13502

This article is protected by copyright. All rights reserved. 


\section{Objective:}

To assess the utility of LP in patients being evaluated for possible SAH in the ED after a negative non-contrast CT scan of the brain, as well as addressing the knowledge gap in regard to rational diagnostics among clinicians

\section{Methods:}

We conducted a retrospective data analysis of patients being evaluated for possible SAH between June 2013 and June 2018 across three emergency departments in Victoria, Australia. A diagnosis of SAH was defined by SAH on CT or an abnormal CSF result in conjunction with positive results on cerebral angiography. A follow-up period of 6 months was chosen.

\section{Results:}

4,407 met inclusion criteria. 397 patients ( $9 \%$ ) were diagnosed with SAH on CT. 388 patients underwent LP and 778 were admitted to hospital for further investigations and management. 2,613 patients discharged from ED without LP remained well at 6 months from their initial presentation, but 230 cases were lost to follow-up. one patient died from haemorrhagic stroke during his third hospitalisation. A single patient demonstrated a true positive LP after a normal CT brain.

\section{Conclusion:}

LP is not required in all patients with suspected subarachnoid haemorrhage who have a negative CT scan. There are defining patient characteristics that can be used to risk stratify patients and may eliminate the need for LP.

Keywords: Subarachnoid haemorrhage, SAH, LP, Lumbar puncture, Headache

\section{Introduction}

Subarachnoid haemorrhage (SAH) is the extravasation of blood into cerebrospinal fluid (CSF). SAH occurs due to mechanical trauma or via rupture of a cerebral aneurysm ${ }^{1}$. The blood irritates the meninges and raises intracranial pressure, causing severe, sudden-onset headache. This is a medical emergency with mortality rates approaching $50 \%$ but can also cause significant neurological morbidity in survivors ${ }^{2}$. It is therefore important to diagnose patients presenting with SAH rapidly to manage life-threatening complications and provide the best chance of recovery. 
$\mathrm{SAH}$ remains a challenging diagnosis to make in the low risk presentation. According to a metaanalysis by Carpenter et al. in $\mathbf{2 0 1 6}^{2}$, the overall SAH rate amongst patients presenting with their worst ever headache that reaches peak intensity within 60 minutes is about $7.5 \%$. Amongst this group of patients, however, there will be a spectrum of risk from high to low.

It is the authors' opinion that there appears to be a knowledge gap amongst clinicians when performing risk assessments for potential SAH presentations and this leads to the over investigation or inappropriate investigation.

There are three potential diagnostic pathways after a negative plain CT brain: lumbar puncture (LP), CT Angiogram (CTA), or no additional workup.

High risk presentations may actually be disadvantaged by a "if CT negative, then perform LP" or "CT $\rightarrow \mathrm{LP}$ " approach, leaving patients with a risk as high as 1.6\% ( 1 in 64). A low risk presentation, by contrast, may have a risk after negative CT alone as low as 1 in 5,000 .

Adopting a standard CT-LP approach without additional rational risk assessment leads to extreme over-investigation of low risk presentations with an increase in harm and potential dismissal of a dangerous diagnosis after serial negative testing in high risk presentations.

This study aims to examine the $C T \rightarrow L P$ pathway to evaluate the utility of this as a routine diagnostic approach in the work-up of patients with potential SAH.

\section{Methods:}

This is a retrospective data analysis of patients presenting to three Monash Health EDs between June 2013 and June 2018 with headache that was evaluated for possible SAH. Monash Health is the largest health network in the state of Victoria, Australia, with an annual emergency department network census of about 200,000 presentations; it comprises three hospitals: Monash Medical Centre (tertiary referral), Dandenong and Casey hospitals (district hospitals).

Two resources were utilised for the data collection:

1. Patients aged 18 years and over, presenting for the first time with a headache were identified by searching the ED electronic medical records (Symphony, EMIS Health, Leads, UK). Cases were analysed to detect if they underwent CTB +/- LP or whether the Neurosurgical team were consulted during their ED stay.

2. Data from the pathology database was retrieved for patients who underwent LP during their ED stay.

The inclusion criteria were: patients who presented for the first time to one of the EDs with a headache who had an initial plain CT. These patients had their records reviewed and the investigation type and results, interventions, and final disposition were recorded.

The exclusion criteria were: patients under 18 and patients who had presented with headaches before the index case. 
To avoid missed cases, all LP episodes from the pathology database across the three EDs during the study period were also entered into a spreadsheet. Cases were compared between the spreadsheets with Structured Query Language (SQL) in Excel, where patient details were matched with the LP time and date. The LP database was hand-searched for errors in LP output, and cases with incomplete results were excluded. Eligible cases were entered into two de-identified Excel spreadsheets and analysed. We assessed inter-rater agreement for 10 percent of cases with a 100 percent inter-rater agreement.

To identify any poor outcomes after discharge, data was obtained from the electronic medical record (EMR) and a 6 months follow-up period was used.

Initially, we considered identifying the clinician's justification for organising a CT, but this was impractical due to resource constraints and the retrospective study design; clinician gestalt was assumed and no specific criteria, such as the Ottawa SAH Rule, were analysed. Searching for the use of any risk stratification methods was also considered but our EMR made this impractical too.

\section{Results:}

4,407 patients met inclusion criteria. 397 ( $9 \%$ ) of these were diagnosed with SAH on plain CT and managed by the neurosurgical team. Of the remaining 4,010 patients with negative $C T$ imaging, 2,844 (70.9\%) were discharged home from the ED without further investigation. Of the 1,166 patients not discharged, 388 underwent LP in the ED and 788 patients were admitted for further investigation and management.

11 (2.8\%) of the 388 patients who had LP demonstrated either red cells or xanthochromia in their CSF (Appendix 1). Only 2 (0.5\% of 388 ) of the LPs were initially deemed actual true positives by the neurosurgical team. The first patient subsequently underwent CTA, DSA, and MRA which were all completely normal. The second patient had red cell counts of 50,580, 43,200, and 51,300 x 106/L, in tubes 1, 2, and 3, respectively. The xanthochromia result demonstrated an oxyhaemoglobin level of 0.073 (0.000-0.020) and a bilirubin level of 0.012 (0.000-0.007). This patient had a small volume of intraventricular blood within the left occipital horn noted on MRI but no aneurysm, vascular malformation, or any other evidence of subarachnoid blood was ever identified by any modality. Both patients were subsequently discharged with outpatient follow up and remained well at the conclusion of follow-up.

We would argue that the first of the two patients did not have a "true positive" LP, given the lack of evidence of haemorrhage or vascular abnormality from any other imaging modality. The second patient, whilst having evidence of a small amount of intraventricular blood, was not a "true positive" in the strictest sense, in that they did not have a SAH, let alone one from an aneurysm.

2,211 patients re-presented to either the ED or outpatient clinics within 6 months from initial discharge with symptoms unrelated to their initial ED presentation. 230 cases $(5.7 \%$ of the 4,010 patients with normal CT scans) were lost to follow-up, owing to a lack of available data (figure 1 ).

Figure 1: Study design flowchart 


\section{Discussion}

From the initial 4,010 patients whose CT brain scans were reported as negative, 388 patients had a LP in our study, there were two cases with a positive LP.

The first patient had an MRI which showed evidence of a small volume of intraventricular blood, but no vascular abnormality ever discovered, i.e. this was not a case of a SAH missed by CT.

The second patient was in a very high risk category, with multiple positive findings on history and examination. This case had a CT scan that was initially reported as negative by a radiology trainee and clearly marked "preliminary". By the time the CSF result was available, the supervising consultant radiologist had written an addendum report that described "high attenuation material in the Sylvian fissures bilaterally, in keeping with a subarachnoid haemorrhage". A subsequent CTA demonstrated, "a $4 \mathrm{~mm}$ aneurysm arising from the anterior communicating artery". This case is discussed further below as we believe a CTA should have followed the patient's CT immediately, rather than diagnosis delayed by waiting for LP results.

Our study compares to the results of a study by Sayer et al from the UK ${ }^{\circ}$ that demonstrated a very low miss rate from negative $\mathrm{CTs}$, even when interpreted by radiologists in-training or qualified general radiologists. Their study had to exclude $15.6 \%$ of the LPs performed as they were uninterpretable or unsuitable for analysis (insufficient sample, exposed to light, blood contamination, incorrect storage, incorrect transport, or sample loss). Of those LPs that they were able to analyse, $15 \%$ were inconclusive, $75.8 \%$ were negative, and $4.8 \%$ were initially considered positive but less than $10 \%$ of these were associated with a vascular abnormality. Their overall LPpositive rate was $0.4 \%$.

Traditionally, the trigger for exclusion of SAH depends on a moderate degree of clinical suspicion and starts with a non-contrast CTB. A negative CT scan is often followed by a LP in patients when the clinician feels the patient has a high pre-test probability. However, this risk is often over-rated by clinicians and a lumbar puncture is an invasive and inaccurate investigation that can cause numerous complications, including infection, epidural haematomata, nerve root injury, spinal headaches, CSF leak, etc. There is a high incidence of false positive LPs, due to the low risk nature of the disease after a negative $C T$, and these put patients at risk of further unnecessary investigations as well as unnecessary admissions.

Carpenter et al published a meta-analysis in $2016^{2}$ which demonstrated a pooled LR+ of 15.23 and a pooled LR- 0.13 for xanthochromia (UK Spectrophotometric). Whilst Carpenter listed a pooled sensitivity of $100 \%$, there were wide confidence intervals (59\% to $100 \%$ ) and the specificity of $95 \%$ ( $93 \%$ to $96 \%$ ) is poor in a low risk population. Computing the sensitivity and specificity backwards from the absolute values of the pooled the Likelihood Ratios, gives a sensitivity of $87.7 \%$ and specificity of $94.2 \%$. When dealing with a low risk group, this means a lot of false positives. When dealing with a high risk group, this means a lot of false negatives. (The equation for computing the sensitivity and specificity from positive and negative likelihood ratios is shown in Appendix 2-6). 
Carpenter demonstrated a weighted average pre-test probability of $7.5 \%$ for $\mathrm{SAH}$ in patients with sudden onset severe headache that reached maximum severity within no more than 60 minutes (the prevalence in our study was $9 \%$ ). This analysis assessed a number of clinical indicators, both historical and on physical examination (table 1), that we believe can be used to modify this baserate to a post-initial-assessment probability and stratify patients into high and low risk categories.

Table 1. Summary of Likelihood Ratios from Carpenter's 2016 meta-analysis²

It is our observation that many clinicians tend to focus only on the positive clinical findings and largely ignore the negative findings when it comes to making their assessment of risk for $\mathrm{SAH}$. Both sets of findings should be used in conjunction and the negative findings are often more numerous, resulting in a significant reduction of risk from the base-rate of $7.5 \%$.

The typical presentation with an abrupt onset headache (maximum severity within 5 minutes), in conjunction with a description of its being the worst headache the patient has ever suffered, but in the absence of any other positive features, may have a post-clinical-assessment risk of no higher than $1.5 \%$, on the basis of the numbers from the meta-analysis by Carpenter et al2. A CT scan in this patient population at 12 hours, where the sensitivity is over $98 \% \%_{11,12}$, will produce a post-negative-CT probability of less than 1 in 3,200 (Figure 2). This is a threshold far below what is tolerated in any other condition for which there are guidelines and where the probability is well understood, e.g. in chest pain and myocardial ischaemia.

Figure 2. Diagnostic Logic

A high risk patient, in contrast, with a post-initial-assessment probability of $80 \%$ (multiple positive and few negative features on history and examination) may have a post-CT risk as high as $7.4 \%$, following a negative scan at 12 hours. Since lumbar puncture in this patient population may miss up to $12.3 \%$ of patients with $\mathrm{SAH}^{2}$ (sensitivity $87.7 \%$ ), a post-LP probability could be as high as $1.4 \%$ (about 1 in 72). If a CT was performed within 6 hours in this high risk patient, they would have posttest risk of missed-SAH of approximately $3.8 \%$ prior to LP and, even after LP, would still have a risk of about $0.5 \%(\sim 1$ in 192). It would seem illogical to be satisfied with a negative $C T$ and a negative LP in a high risk patient but to be unsatisfied with only a negative CT in a low risk patient, when the latter patients are at much lower risk than the former ones.

The post-test threshold is up to the discretion of the individual clinician, in conjunction with informed decision making with the patient, and there is literature available to guide them such as a 2016 paper by Taylor et al ${ }_{3}$ that specifically calculated a test-threshold for LP after a negative CT. The vast majority of presentations are likely to need no more than a CT scan. High risk patients who are missed on CT may also be missed on LP and an alternative approach with CTA may be warranted in these much less common presentations. 
A "traumatic tap" can distort the sample. Traditionally, in order to distinguish between a traumatic tap and a true $\mathrm{SAH}$, the three-tube test of progressively decreasing red cells is used in combination with the absence of xanthochromia. A traumatic tap with a significant number of red cells can leave that third tube heavily contaminated, leading to an "equivocal" result. This is likely to be, at least in part, why the LP has a lower sensitivity than might be expected. Performing an LP earlier may miss xanthochromia due to the fact that it takes time to form.

By definition, xanthochromia is the yellow discolouration of the cerebrospinal fluid (CSF), indicating the presence of bilirubin, and is used by some to differentiate in vivo haemorrhage from a traumatic LP. In contrast to CT, CSF xanthochromia is present in most patients up to 2 weeks post-ictus and is still present in $70 \%$ of patients at 3 weeks 7.8 . A minimum period for CSF bilirubin detection is $12 \mathrm{~h}$ post-ictus?. Thus, the detection of bilirubin (xanthochromia) in CSF appears to be the test of choice in delayed high risk presentations. Spectrophotometry of CSF in the visible region is, in general, considered more sensitive than visual examination, with peaks at 415 and $\sim 440-460 \mathrm{~nm}$ indicating the presence of haemoglobin $(\mathrm{Hb})$ and bilirubin, respectively ${ }^{78}$.

As in almost all situations though, as the sensitivity of a test increases, specificity falls and so there is a trade of between false negatives and false positives. The problem with this test is that it is not known which CSF bilirubin absorbance indicates a clinically significant bleed, hence the reduction in specificity.

In our study, all but one positive LPs were identified as false positives (a total of 11 patients) for aneurysmal SAH. These patients underwent further investigation and had increased lengths of stay in hospital. Similar to previous studies by Perry et al., which recommend against the routine use of LP after a negative CT scan performed early ${ }^{3,4}$, our study emphasises the drive to improve the clinical risk stratification and workup of patients with potential for SAH.

\section{Presentation time}

The result of a study published by the BMJ in 2011 by Perry et al 4 is well known to the community in that it showed CT is very sensitive within 6 hours, with the caveats that it was performed with a modern multi-row detector third generation scanner with thin slices and that the scans were interpreted by a qualified radiologist. What we found particularly interesting in this study is that they also showed that the risk of SAH in patients presenting within 6 hours was $12.7 \%$ whilst the risk of patients presenting over 6 hours was $5.46 \%$. This represents an increase in odds ratio by 2.32 if a patient presents within 6 hours or a reduction in odds ratio by 0.43 if a patient presents after 6 hours. These odds ratios are restricted to the patients investigated in their study population, but similar odds have been observed elsewhere.

A trial in 2012, performed in a Dutch Tertiary Hospital, looked at the time dependent characteristics of patients being worked up for $\mathrm{SAH}$, and found that more than half of the patients with a diagnosis of SAH presented to their centre within 6 hours of symptom onset.

It is our hypothesis that the falling sensitivity of CT over the first 12-24 hours may be offset by a fall in risk of SAH and this time-to-presentation variable may be an area that warrants further exploration in future trials. 
Our network is moving towards a risk stratification approach where low risk patients are excluded from further investigation for SAH after a normal CT and high risk patients are followed up with a CT angiogram. This latter group is a small in our population and the CTA is pre-planned and performed regardless of non-contrast CT result since a positive CT would be followed by a CTA anyway.

Different patient populations in different regions of the world will present at different stages of their symptoms so clinicians should consider their local population when considering the utility of the CT-alone approach.

\section{A suggested approach to a possible Decision Rule}

The authors would like to suggest a risk stratification tool that is based on the data from the metaanalysis by Carpenter et al.

Whilst our approach is more complicated than a score like the Well's Criteria used in the workup for pulmonary embolus, it is our opinion that this is the direction in which diagnostics should progress.

Within our local communities, we need to establish an agreed acceptable miss-rate. This can be very difficult to establish in different populations with different litigation rates and expectations but there is always a miss rate. As clinicians target lower and lower risk patients, the intervention use increases exponentially and therefore the cost to the population. Perhaps more importantly, though, the rate of harm from unnecessary investigations and the treatment of patients with falsepositive tests rises too.

A test-threshold can be calculated for any given diagnosis when the harm of missing it (or the benefit from intervention) is considered along with the harm from testing and the harm from treating false-positives. These metrics will vary from population to population and from service to service. The outcomes used may not be equivalent such as death vs transient complication, so it is not always possible to compute a threshold. The serious negative outcomes from investigating patients for SAH with a LP would be epidural abscess or haematoma with permanent disability in addition to the harms from intervention in patients with an incidental aneurysm discovered that may never have ruptured but get an intervention that leads to disability or death. A threshold equation is not further covered here.

Starting with an agreed acceptable miss-rate, such as $<1$ in 1,000 , we can then compute backwards from our desired post-CT probability to what our post-assessment probability threshold should be. If we take the lowest sensitivity of CT at $98 \%$ within 12 hours, the negative likelihood ratio of CT is 0.02 . At a desired post-test risk of $<1$ in 1,000 , this means a pre-CT risk of $<4.8 \%$ would be tolerated within 12 hours. At a sensitivity of $99 \%$ within 6 hours, with a likelihood ratio of 0.01 , a pre-CT risk of $<9.1 \%$ would be tolerated. This ignores any potential to reduce the patient's pre-test risk by a delay to presentation.

If we assess a patient as having a pre-test probability of SAH less than $4.8 \%$ at $6-12$ hours or less than $9.1 \%$ at $0-6$ hours, a negative CT would represent a risk of less than 1 in 1,000 of a missed SAH. 
The next question a clinician should consider is how they estimate a patient's pre-CT risk. From the data in the meta-analysis by Carpenter ${ }^{2}$, we can chain together the independent likelihood ratios and produce a score threshold that modifies the expected base-rate of $\sim 7.5 \%$ to the desired pre-CT threshold for the patient. If we choose a pre-CT threshold $4.8 \%$ (post-CT risk of $<1$ in 1,000 within 12 hours), we can compute the required product of Carpenter's likelihood ratios or "combination LR" (cLR) to be $<0.617$ (The details of this calculation are shown in the Appendix 2-4). For CT occurring within 6 hours at a sensitivity of $>99 \%$, the CLR needs to be $<1.233$ to give a post-CT risk of $<1$ in 1,000 .

From here, it is just a matter of multiplying together the likelihood ratios from Carpenter's metaanalysis and seeing whether the CLR is greater or less than 0.617 (for CT 6-12 hours) or 1.233 (for CT o-6 hours).

Individual centres should choose their own post-CT threshold and therefore compute their own CLR for presentations within 6 or 12 hours. Beyond these timeframes, it becomes less certain as the sensitivity of $\mathrm{CT}$ is less well known.

\section{Worked example (LRs in brackets)}

A patient presents with their worst headache ever (1.25) which reached maximum intensity within a few minutes (1.34) and there is ongoing nausea (1.15) but no vomiting (0.52) and no meningism (0.78); the headache did not come on during exertion (0.88), there was no loss of consciousness (0.91), and they are able to walk (0.85). Multiplying these LRs together give a cLR of 0.53 .

Starting with $7.5 \%$ as a baseline probability and modifying that with the CLR of 0.53 , a pre-CT or post-assessment probability is $\sim 4.1 \%$.

If the $\mathrm{CT}$ is performed between $6-12$ hours, at a sensitivity of $98 \%$, the post-CT probability of a false negative is $<1$ in 1,160 . This is a margin of risk far lower than Emergency Physicians tolerate in other conditions. Individual clinicians may, of course, choose an even lower margin, if that is their preference.

The only case where there was a "true positive" LP in a patient with a "normal CT" in our study was the patient mentioned earlier who had a preliminary $C T$ report by a radiology trainee (clearly marked at the time as such) that was subsequently reported by a consultant radiologist as demonstrating a SAH. This patient had multiple risk factors for SAH, namely: thunderclap, worst headache, onset during exertion, neck pain, syncope, nausea, vomiting. Using Carpenter's likelihood ratios, we would estimate his pre-CT probability of having a SAH as being over $80 \%$ and that, had he had a negative CT within 6 hours (at a sensitivity of $99 \%$ ) and a negative LP, his postnegative-tests risk would have been about $0.8 \%$ or about 1 in 124 . To us, this is an unacceptably high risk for SAH but many clinicians would drop their suspicion for the diagnosis with both a negative CT and LP. Fortunately for this patient, the CT was subsequently reported correctly and prior to discharge; the positive LP was just additional information. Adopting a CT then CTA approach for high risk patients such as this patient would have identified the aneurysm responsible for the SAH much earlier. 


\section{Limitations}

We are unlikely to have failed to capture all patients presenting to our network with headache who had CT imaging over the study period but there were other limitations.

Due to the retrospective study design and the large number of patients being reviewed, we only had the resources to track representations and because of this, combined with limited ethics approval, we were not able to contact patients who had no further presentations to our network after being discharged after the index visit. It is unlikely that there were patients missed by this approach, however repeat presentations are generally to the same health network in our region as our network covers a very large geographical area. In addition, hospitals generally notify each other of unexpected deaths following recent discharges. This makes attrition bias unlikely but not impossible.

We were unable to identify any specific difficulties encountered during LP that may have skewed our data. A greater number of traumatic taps or those done earlier than the time required for xanthochromia to develop may have limited the accuracy of LP results. We did not have the ability to control for the level of expertise in performing LP as, in general, it is the junior medical staff (1-7 years experience) who perform them in our network. Traumatic LPs are therefore not uncommon, so their specificity is likely to be reduced.

We were unable to record time from symptom onset to presentation and we believe this affects the probability of $\mathrm{SAH}$, with delayed presentations having lower risk. This is concept is covered in the discussion section.

The generalisability of the results of our study beyond our network and patient population may limit the external validity of our study.

\section{Conclusion}

Research in the last 8 years within our three Emergency Departments has shown that the sensitivity of $C T$, when performed early from the onset of symptoms, is sufficiently high enough to make a follow-up LP unnecessary in the majority of presentations. Our study adds to this in that no true positive LPs (for SAH) were found in any patients after a final report of a negative non-contrast CTB, proposing a low diagnostic necessity in this local cohort.

We suggest clinicians adopt an approach that takes into account both the positive and negative clinical findings in a presentation to calculate a pre-CT risk and thence a post- $C T$ risk. $C T$ is very sensitive up to 24 hours, even when reported by non-specialist neuroradiologists, and will exclude the majority of patients with a genuine SAH.

High risk patients should have a CTA instead of a LP as the post-LP risk of a false negative is potentially high. The majority of high risk patients will be detected on plain CT and this will typically be followed by CTA to identify the aneurysm responsible. It therefore stands to reason that all 
genuine high risk patients should have a CT and CTA as a matter of standard protocol, whilst low risk patients should have only a plain CT.

Determination of risk can be done by referring to the increasingly large body of literature out there on the likelihood ratios for presenting features, most recently summarised by Carpenter et al in $2016^{2}$.

Further prospective studies should be conducted with a more thorough follow up plan to emphasise these findings.

\section{Authorship statement}

The authors have no conflicts of interest to declare and were not recipients of research funding relevant to this study. The authors are in agreement with the contents of the manuscript.

\section{References}

1. Suarez, J.I., R.W. Tarr, and W.R. Selman, Aneurysmal subarachnoid hemorrhage. N Engl J Med, 2006. 354(4):387-96.

2. Carpenter, C.R., et al., Spontaneous Subarachnoid Hemorrhage: A Systematic Review and MetaAnalysis Describing the Diagnostic Accuracy of History, Physical Exam, Imaging, and Lumbar Puncture with an Exploration of Test Thresholds. Academic emergency medicine : official journal of the Society for Academic Emergency Medicine, 2016. 23(9):963-1003.

3. Perry, J.J., et al., Validation of the Ottawa Subarachnoid Hemorrhage Rule in patients with acute headache. CMAJ, 2017. 189:E1379-85.

4. Perry, J.J., et al., Sensitivity of computed tomography performed within six hours of onset of headache for diagnosis of subarachnoid haemorrhage: prospective cohort study. BMJ, 2011. 343:D4277.

5. Backes, D., et al., Time-dependent test characteristics of head computed tomography in patients suspected of nontraumatic subarachnoid hemorrhage. Stroke, 2012. 43(8):2115-9.

6. Edlow, J.A. and J. Fisher, Diagnosis of subarachnoid hemorrhage: time to change the guidelines? Stroke, 2012. 43(8):2031-2.

7. Van Gijn J, van Dongen KJ. Time course of aneurysmal haemorrhage on computed tomograms. Neuroradiology 1982;23:153-156.

8. Vermuelen M, Hasan D, Blijenberg BG, Hijdra A, Van Gijn J. Xanthochromia after subarachnoid haemorrhage needs no revisitation. J Neurol 1989;52:826-828.

This article is protected by copyright. All rights reserved. 
9. Backes D et al; Stroke. 2012 Aug;43(8):2115-9

10. Sayer et al. The use of LP to rule out SAH after a normal CT. Academic Emergency Medicine $\bullet$ Nov 2015, Vol. 22, No. 11

11. Morgenstern LB, et al. Worst headache and subarachnoid hemorrhage. Ann Emerg Med. 1998; 32:297-304.

12. Sidman RE et al.. Subarachnoid hemorrhage diagnosis: lumbar puncture is still needed when the computed tomography scan is normal. Acad Emerg Med. 1996;3:827-831.

13. Taylor, R. A. Et al. Determination of a Testing Threshold for Lumbar Puncture in the Diagnosis of Subarachnoid Hemorrhage after a Negative Head Computed Tomography: A Decision Analysis.

Academic Emergency Medicine, 23(10), 1119-1127. 
Figure legends:

Figure 1: Study design flowchart.

Table 1. Summary of Likelihood Ratios from Carpenter's 2016 meta-analysis ${ }^{2}$

Figure 2. Diagnostic Logic

Table 2. Imaging and CSF with disposition

This article is protected by copyright. All rights reserved. 
Appendix 1

Table 2: Imaging and CSF with disposition

This article is protected by copyright. All rights reserved. 


\section{Appendix 2}

1. Odds Ratios and Probabilities

Likelihood ratios are ratios, not probabilities and therefore the base-rate probability must be converted to an odds ratio. Odds ratios don't mean much to people and should be converted back to probabilities.

1. Converting probabilities to odds ratios

$$
\begin{aligned}
& \text { odds ratio }=\frac{\text { probability }}{1-\text { probability }} \\
& \text { e.g. } \frac{7.5 \%}{1-7.5 \%}=0.081
\end{aligned}
$$

2. Converting odds ratios to probabilities

$$
\begin{gathered}
\text { probability }=\frac{\text { odds ratio }}{1+\text { odds ratio }} \\
\text { e.g. } \frac{0.25}{1+0.25}=20 \%
\end{gathered}
$$

2. Likelihood ratios 
Positive likelihood ratios refer to when a test is positive or the characteristic is present and negative likelihood ratios the converse. They are ratios of patients with the disease of interest to those without.

1. Positive Likelihood Ratio

$$
\begin{aligned}
& \mathrm{LR}^{+}=\text {feature present } \frac{\text { with disease }}{\text { without disease }} \\
& \mathrm{LR}^{+}=\frac{\text { sensitivity }}{1-\text { specificity }}
\end{aligned}
$$

2. Negative Likelihood Ratio

$$
\begin{aligned}
& \mathrm{LR}^{+}=\text {feature absent } \frac{\text { with disease }}{\text { without disease }} \\
& \mathrm{LR}^{+}=\frac{1-\text { sensitivity }}{\text { specificity }}
\end{aligned}
$$

3. "Acceptable" pre-test probability

Once an agreed acceptable miss-rate is established, such as $<1$ in 1,000 , we can then compute backwards from our desired post-test probability to what our post-assessment probability threshold should be. If our test is $98 \%$ sensitive and $100 \%$ specific, it's negative likelihood ratio $(L R-)$ is 0.02 .

Since: post - test odds $\left(\frac{1}{1000}\right)=$ post - assessment odds $(? ?) \times \mathrm{LR}^{-}(0.02)$

Therefore, pre - test odds $=\frac{1}{1000} \div 0.02=0.05$ (4.8\% as a probability)

4. Combined Likelihood Ratio (cLR)

Now that we have a pre-test odds, we can work out what our cLR should be. The overall rate of $\mathrm{SAH}$ in the meta-analysis by Carpenter et al was $7.5 \%$ with a pre-assessment odds of 0.081 . 
1. Presentations between 6-12 hours

Since: pre - test odds $(0.05)=$ pre - assessment odds $(0.081) \times \operatorname{cLR}^{-}(? ? ?)$

Therefore, $\mathrm{cLR}=\frac{\text { pre }- \text { test odds }}{\text { pre }- \text { assessment odds }}=\frac{0.05}{0.081}=0.617$

With a CLR of 0.617 for patients having a CT between $6-12$ hours, a negative scan produces a post-test probability of $<1$ in 1,000 .

2. Presentations between 6-12 hours

Since: pre - test odds $=\frac{1}{1000} \div 0.01=0.1(9.1 \%$ as a probability $)$

And: pre - test odds $(0.1)=$ pre - assessment odds $(0.081) \times \operatorname{cLR}^{-}(? ? ?)$

Therefore, $\mathrm{cLR}=\frac{\text { pre }- \text { test odds }}{\text { pre }- \text { assessment odds }}=\frac{0.1}{0.081}=1.233$

With a CLR of 1.233 for patients having a CT within 6 hours, a negative scan produces a post-test probability of $<1$ in 1,000 .

\section{Carpenter's Likelihood Ratios}

Table 3

Worked example - expanded from main narrative

A patient presents with their worst headache ever (1.25) which reached maximum intensity within 5 minutes (1.34) and there is ongoing nausea (1.15) but no vomiting (0.52), no meningism (0.78); the headache did not come on during exertion (0.88), there was no loss of consciousness (0.91), and they are able to walk (0.85).

\section{$1.25 \times 1.34 \times 1.15 \times 0.52 \times 0.78 \times 0.88 \times 0.91 \times 0.85=0.53$}

Starting with $7.5 \%$ as a baseline probability and modifying that with the CLR of 0.53 , a pre-CT or post-assessment probability is $4.1 \%$.

This article is protected by copyright. All rights reserved. 


$$
\frac{7.5}{92.5} \times 0.53=0.043(\text { odds })=4.1 \%(\text { probability })
$$

If the $\mathrm{CT}$ is performed between $6-12$ hours, at a sensitivity of $98 \%$, the post-CT probability of a false negative is $<1$ in 1,160 . This is a margin of risk far lower than Emergency Physicians tolerate in other conditions. Individual clinicians may, of course, choose an even lower margin, if that is their preference.

6. Sensitivity and Specificity from Positive and Negative Likelihood Ratios

$$
\text { Sensitivity }=\frac{L R^{+}\left(1-L R^{-}\right)}{L R^{+}-L R^{-}} \quad \text { Specificity }=\frac{L R^{+}-1}{L R^{+}-L R^{-}}
$$

Example: Carpenter's pooled LRs for LP accuracy

$L R+15.23$

LR- 0.13

Sensitivity: $87.7 \%$

Specificity: $94.2 \%$

This article is protected by copyright. All rights reserved. 


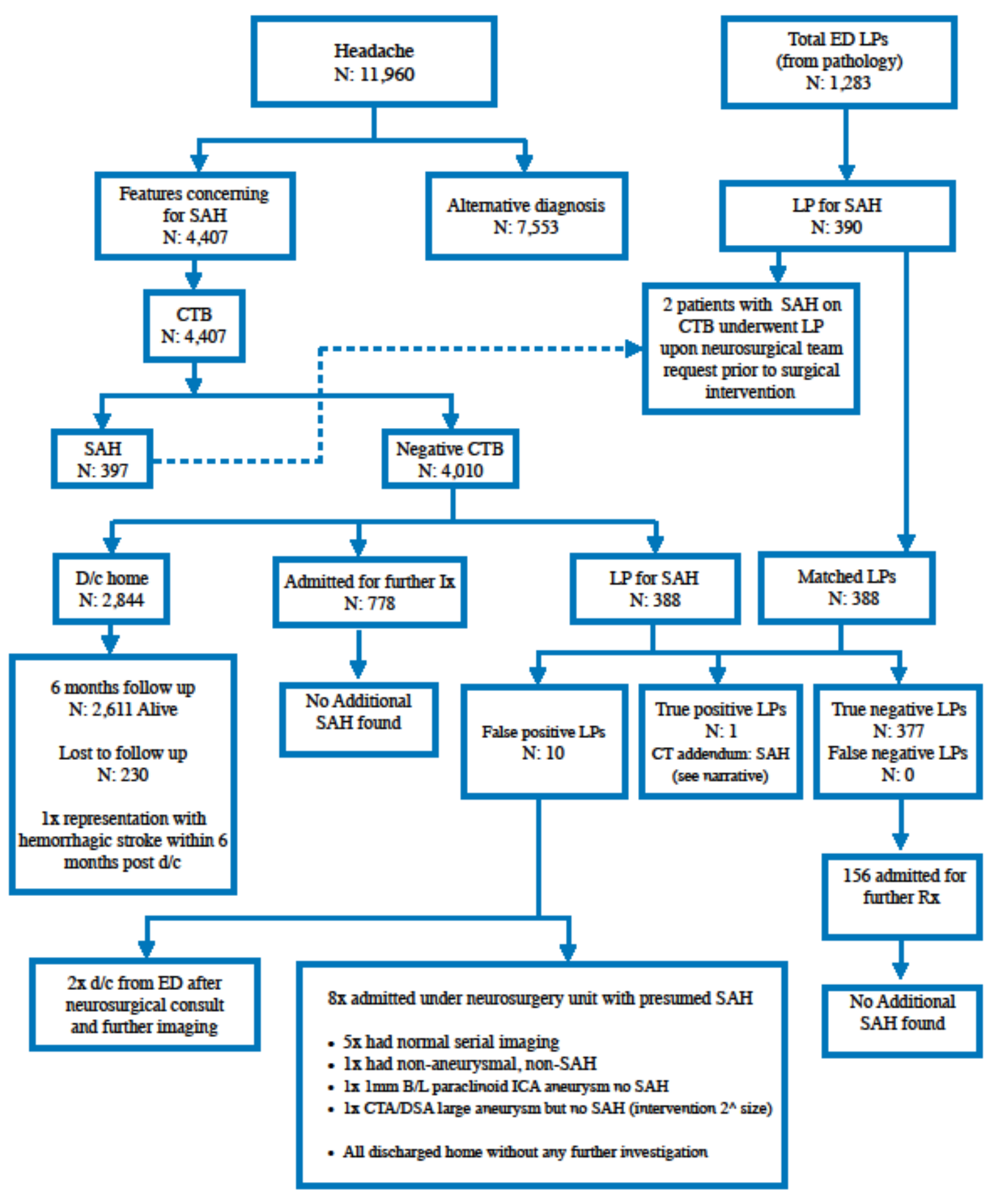

Figure 1: Study design flowchart. 


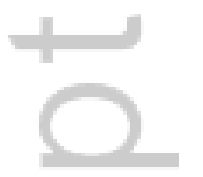




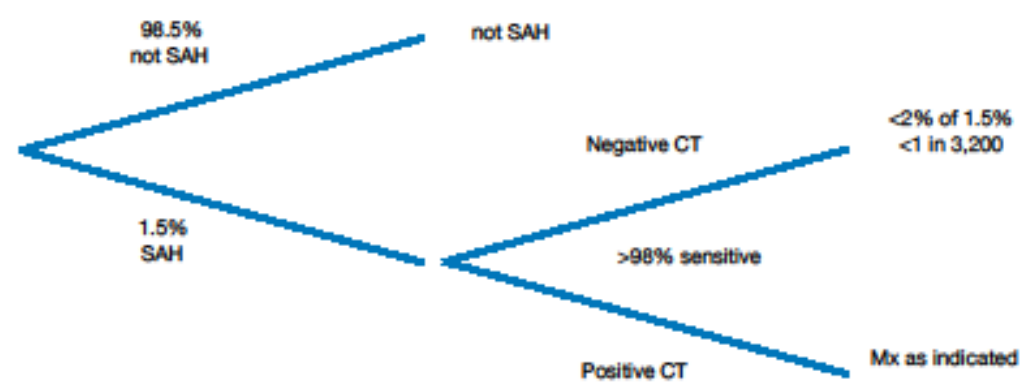

Figure 2. Diagnostic Logic

This article is protected by copyright. All rights reserved. 


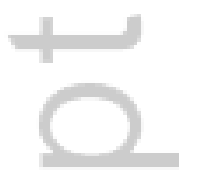




\begin{tabular}{|lcc|}
\hline Historical features & LR+ & LR- \\
Worst headache & 1.26 & 0.24 \\
Thunderclap & 1.34 & 0.74 \\
Exertion at oneet & 1.70 & 0.88 \\
Onset during intercourse & 1.20 & 1.00 \\
Losa of consciousness & 1.87 & 0.91 \\
Blurred viaion & 3.14 & 0.85 \\
Nausea & 1.16 & 0.74 \\
Examination features & & \\
Meningiam & & 0.78 \\
Vorniting & 6.69 & 0.62 \\
Unable to walk & 1.92 & 0.85 \\
Altered mental status & 2.36 & 0.87 \\
Focal neuro deficit & 2.18 & 0.81 \\
\hline
\end{tabular}

Table 1. Summary of Likelihood Ratios from Carpenter's 2016 meta-analysis ${ }^{2}$

This article is protected by copyright. All rights reserved. 


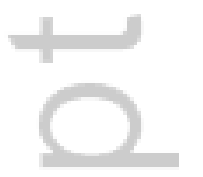


Table 2

Imaging and CSF Results with Dispositions

\begin{tabular}{|c|c|c|c|c|c|c|c|c|c|c|c|c|}
\hline & 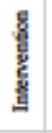 & $\cdot$ & $\cdot$ & $\mid \begin{array}{l}\frac{8}{8} \\
5 \\
5 \\
5\end{array}$ & $\cdot$ & $\cdot$ & \begin{tabular}{|l|} 
\\
5 \\
5 \\
2 \\
5 \\
5
\end{tabular} & . & $\cdot$ & . & $\cdot$ & . \\
\hline & $\begin{array}{l}8 \\
8 \\
8\end{array}$ & $\frac{8}{2}$ & $\frac{8}{6}$ & $\begin{array}{l}0 \\
\frac{1}{6} \\
z\end{array}$ & $\frac{8}{6}$ & $\frac{0}{6}$ & $\frac{E}{8}$ & $\frac{8}{2}$ & $\frac{\pi}{2}$ & $\frac{8}{2}$ & 昜圆 & 噌国 \\
\hline & 9 & $\begin{array}{l}+ \\
\frac{5}{2} \\
\frac{8}{2}\end{array}$ & $\begin{array}{l}+ \\
\stackrel{8}{3}\end{array}$ & $\begin{array}{l}+ \\
\frac{8}{2}\end{array}$ & $\stackrel{+}{8}$ & $\begin{array}{l}+ \\
\stackrel{8}{8}\end{array}$ & $\begin{array}{l}+ \\
t \\
\underline{E}\end{array}$ & $\begin{array}{l}+ \\
\frac{8}{2}\end{array}$ & $\frac{8}{2}$ & $\begin{array}{l}+ \\
\frac{8}{8} \\
\frac{8}{2}\end{array}$ & $\stackrel{+}{8}$ & $\begin{array}{l}+ \\
\frac{8}{2}\end{array}$ \\
\hline \multirow{4}{*}{ 量 } & 鄫 & 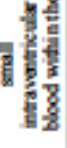 & $z$ & ' & $z$ & $z$ & $z$ & $z$ & $z$ & $z$ & $\cdot$ & $\cdot$ \\
\hline & 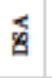 & . & $\cdot$ & 8瓷 & $\cdot$ & . & 焉 & 8荡 & $\cdot$ & . & . & . \\
\hline & $\widehat{ย}$ & $z$ & $z$ & 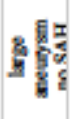 & $z$ & $z$ & そ气 & 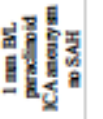 & $z$ & z & z & z \\
\hline & 兒 & $z$ & $z$ & $z$ & $z$ & $z$ & 量数 & $z$ & $z$ & $z$ & z & z \\
\hline \multirow{4}{*}{ 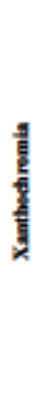 } & s & + & $\cdot$ & + & $\cdot$ & $\cdot$ & + & + & . & + & . & . \\
\hline & $\frac{5}{\frac{1}{1}}$ & + & + & + & + & + & + & + & + & + & + & . \\
\hline & 童变 & ฮี & 8 & 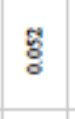 & 8 & పे & 형 & $\overline{\mathrm{g}}$ & 8 & $\circ$ & 8 & 8 \\
\hline & 8 & $\mathrm{~g}$ & 8 & $\frac{\text { t }}{6}$ & 8 & 형 & $\frac{\pi}{6}$ & हैं & ః & 향 & 8 & $\overline{\mathrm{s}}$ \\
\hline \multirow{4}{*}{ 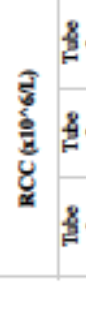 } & 童m & $\frac{8}{m}$ & $\circ$ & 奉 & $\mathrm{H}$ & $\mathscr{A}$ & 骨 & 品 & శ్ళ్రి & $\not$ & $\circ$ & 鰠 \\
\hline & \&्ष & $\underset{7}{\stackrel{9}{7}}$ & $\circ$ & 辛 & $\mathscr{A}$ & 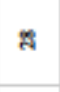 & 悹 & ส్తి & $\underline{8}$ & $\approx$ & A & \& \\
\hline & 浐- & \% & $\circ$ & 莑 & $\$$ & $q$ & 务 & : & 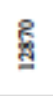 & 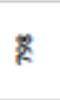 & $\Phi$ & 8 \\
\hline & & - & $A$ & $m$ & + & $n$ & $\bullet$ & 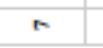 & $\infty$ & $a$ & ؟ & $=$ \\
\hline
\end{tabular}

This article is protected by copyright. All rights reserved. 


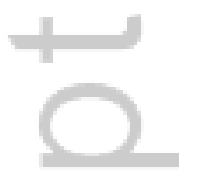


Table 3. Carpenter's Likelihood Ratios

\begin{tabular}{|lrr|}
\hline Historical features & LR+ & LR- \\
Worst headache & 1.25 & 0.24 \\
Thunderclap & 1.34 & 0.74 \\
Exertion at oneet & 1.70 & 0.88 \\
Oneet during intercouree & 1.20 & 1.00 \\
Lose of coneciouenese & 1.87 & 0.91 \\
Blurred vision & 3.14 & 0.85 \\
Nausea & 1.15 & 0.74 \\
\hline
\end{tabular}

\begin{tabular}{|lrr|} 
Examination features & LR + & LR- \\
Meningiem & 6.59 & 0.78 \\
Vomiting & 1.92 & 0.52 \\
Unable to walk & 2.36 & 0.85 \\
Altered mental status & 2.18 & 0.87 \\
Focal neuro deficit & 3.26 & 0.81 \\
\hline
\end{tabular}

This article is protected by copyright. All rights reserved. 


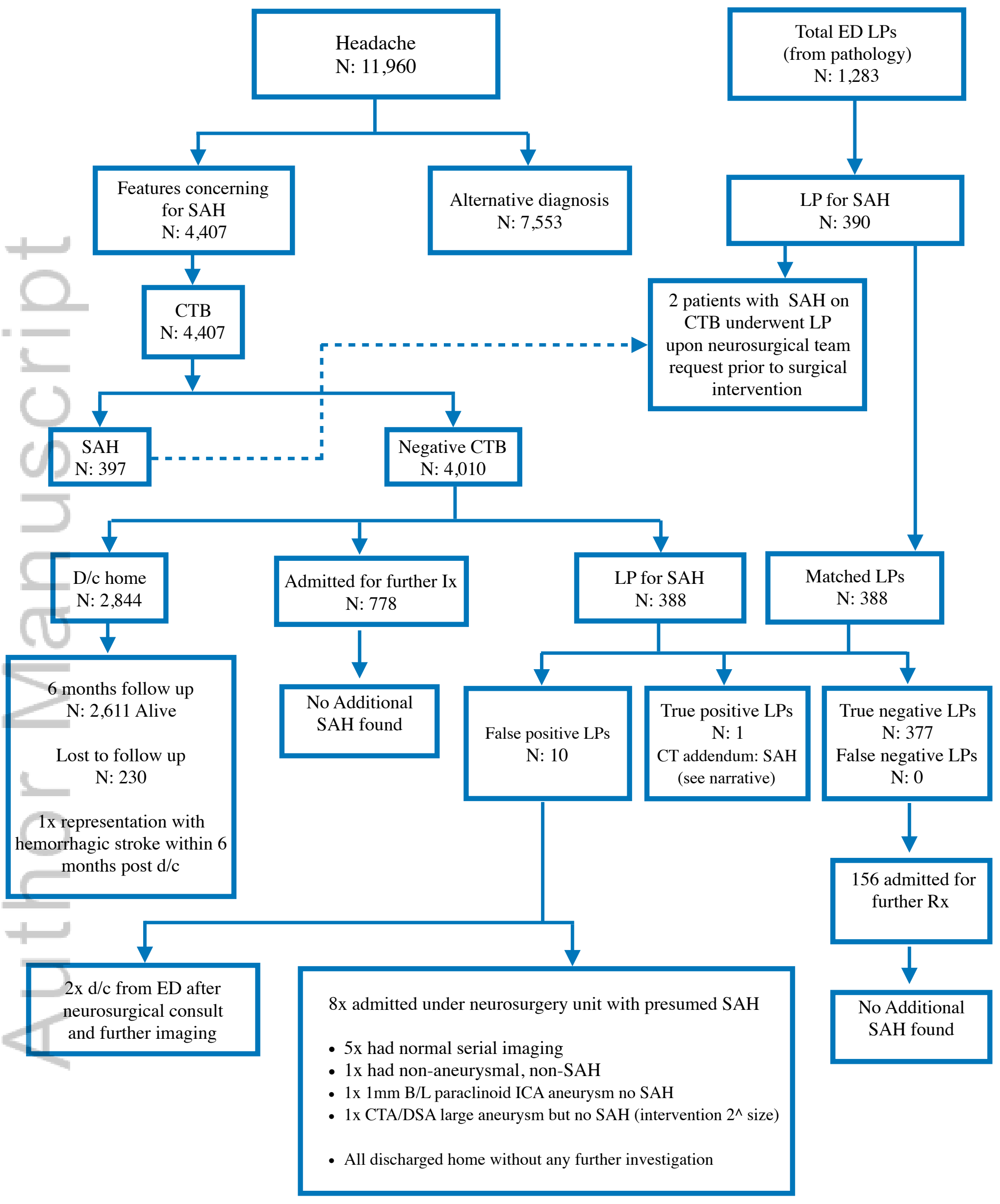

Figure 1: Study design flowchart. 


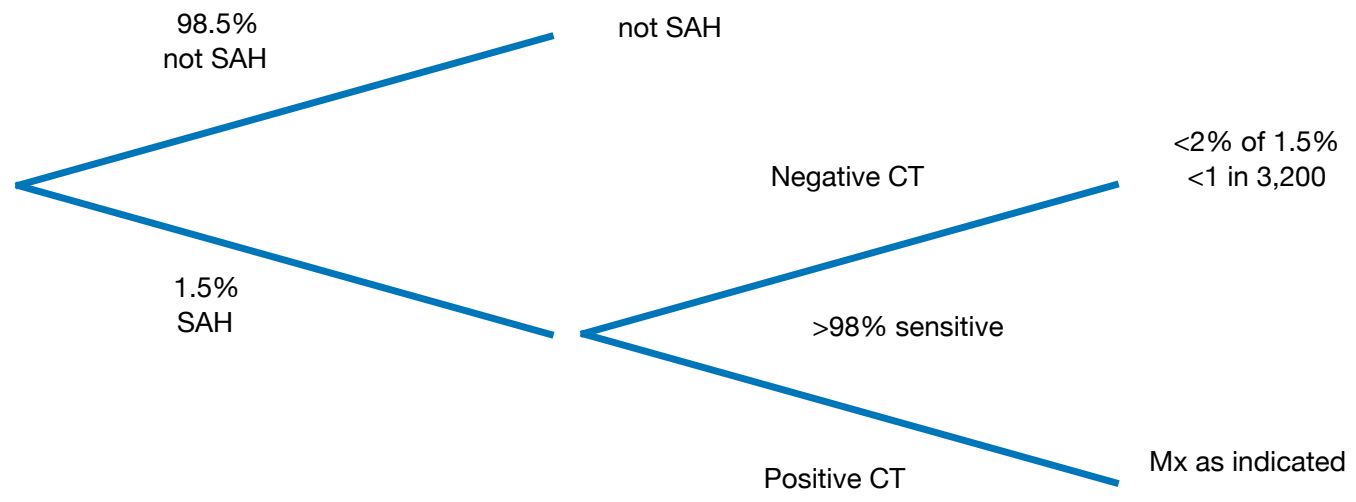

Figure 2. Diagnostic Logic

This article is protected by copyright. All rights reserved. 


\begin{tabular}{|lcc|}
\hline Historical features & LR+ & LR- \\
Worst headache & 1.25 & 0.24 \\
Thunderclap & 1.34 & 0.74 \\
Exertion at onset & 1.70 & 0.88 \\
Onset during intercourse & 1.20 & 1.00 \\
Loss of consciousness & 1.87 & 0.91 \\
Blurred vision & 3.14 & 0.85 \\
Nausea & 1.15 & 0.74 \\
Examination features & & \\
Meningism & 6.59 & 0.78 \\
Vomiting & 1.92 & 0.52 \\
Unable to walk & 2.36 & 0.85 \\
Altered mental status & 2.18 & 0.87 \\
Focal neuro deficit & 3.26 & 0.81 \\
\hline
\end{tabular}

Table 1. Summary of Likelihood Ratios from Carpenter's 2016 meta-analysis²

This article is protected by copyright. All rights reserved. 
Table 2

Imaging and CSF Results with Dispositions

\begin{tabular}{|c|c|c|c|c|c|c|c|c|c|c|c|c|}
\hline & 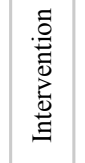 & ' & I & 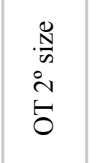 & ' & ' & 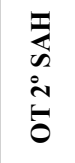 & ' & ' & ' & ' & ' \\
\hline & 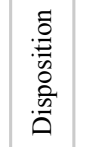 & 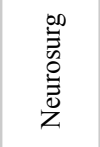 & 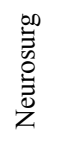 & 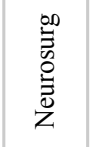 & $\begin{array}{l}\text { on } \\
\bar{z} \\
\overline{0} \\
\bar{\Xi} \\
z \\
z\end{array}$ & $\begin{array}{l}\text { on } \\
\bar{\Xi} \\
\overline{0} \\
\bar{\Xi} \\
\bar{z}\end{array}$ & 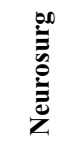 & 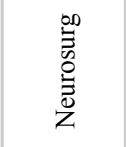 & 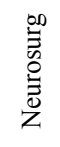 & $\begin{array}{l}\text { on } \\
\text { 产 } \\
\text { 产 } \\
\text { ż }\end{array}$ & 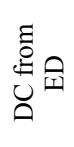 & 莒 \\
\hline & $\overrightarrow{3}$ & $\begin{array}{l}+ \\
+ \\
\frac{0}{0} \\
\frac{\pi}{5}\end{array}$ & $\begin{array}{l}+ \\
0 \\
\frac{0}{0} \\
\frac{0}{5}\end{array}$ & $\begin{array}{l}+ \\
0 \\
\frac{0}{0} \\
\frac{w}{5}\end{array}$ & $\begin{array}{l}+ \\
+ \\
\frac{0}{0} \\
\frac{\sigma}{5}\end{array}$ & 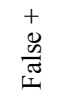 & $\stackrel{+}{\stackrel{D}{g}}$ & 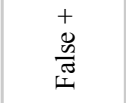 & $\begin{array}{l}+ \\
+ \\
\frac{D}{0} \\
\frac{\pi}{\tilde{E}}\end{array}$ & 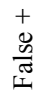 & $\begin{array}{l}+ \\
+ \\
\frac{0}{0} \\
\frac{D}{5}\end{array}$ & 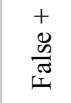 \\
\hline \multirow{4}{*}{ 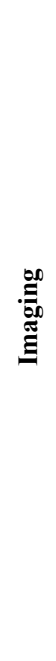 } & $\vec{\xi}$ & 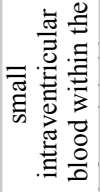 & z & & z & $\mathrm{z}$ & $\mathbf{z}$ & z & z & z & ' & ' \\
\hline & 艿 & ' & ' & $\stackrel{\square}{\stackrel{T}{厶}}$ & ' & ' & $\sum_{n}^{\pi}$ & 焉 & ' & ' & ' & ' \\
\hline & $\overleftrightarrow{E}$ & z & z & 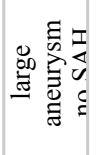 & z & $\mathrm{z}$ & 岁离 & 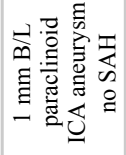 & $\mathrm{z}$ & z & z & z \\
\hline & $\stackrel{n}{\bullet}$ & z & z & z & z & z & 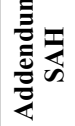 & z & $z$ & z & z & z \\
\hline \multirow{4}{*}{ 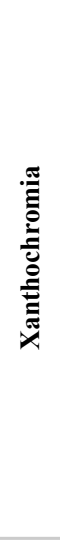 } & 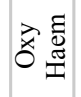 & + & ' & + & ' & ' & + & + & ' & + & ' & ' \\
\hline & 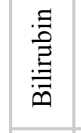 & + & + & + & + & + & + & + & + & + & + & ' \\
\hline & 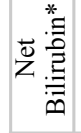 & $\stackrel{?}{\square}$ & ठे & ठิ & ठे. & $\stackrel{\infty}{\circ}$ & $\underset{\check{\Xi}}{\tilde{\delta}}$ & $\begin{array}{l}\bar{n} \\
0 \\
0\end{array}$ & ڤे & 0 & $\hat{8}$ & $\begin{array}{l}0 \\
\stackrel{0}{0}\end{array}$ \\
\hline & 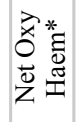 & $\stackrel{\text { }}{0}$ & 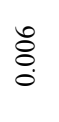 & $\stackrel{\stackrel{I}{I}}{\circ}$ & $\stackrel{0}{0}$ & $\begin{array}{l}\text { ठั } \\
\stackrel{0}{0}\end{array}$ & $\frac{⿱}{5}$ & 草 & $\stackrel{m}{a}$ & 草 & $\stackrel{\infty}{0}$ & $\bar{\circ}$ \\
\hline \multirow{3}{*}{ 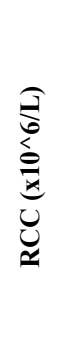 } & $\oslash_{m}$ & $\begin{array}{l}8 \\
\stackrel{m}{n} \\
i\end{array}$ & 0 & $\begin{array}{l}\text { 圭 } \\
\neq\end{array}$ & $N$ & $\stackrel{\sim}{\sim}$ & 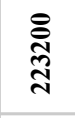 & $\frac{n}{m}$ & 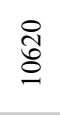 & i & 0 & 离 \\
\hline & $\oiint_{N}$ & $\begin{array}{l}8 \\
\text { N } \\
\text { f }\end{array}$ & 0 & 夆 & $i$ & $\stackrel{\infty}{\sim}$ & 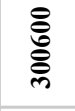 & స్తి & $\begin{array}{l}0 \\
0 \\
0 \\
0\end{array}$ & $\infty$ & $N$ & ๙্ণ \\
\hline & $\overbrace{Z}^{0}-$ & $\begin{array}{l}8 \\
\text { n. } \\
\hat{n}^{2}\end{array}$ & 0 & $\begin{array}{l}\text { 夆 } \\
\text { 去 }\end{array}$ & $\infty$ & $\mathcal{F}$ & $\frac{\stackrel{8}{+}}{\stackrel{+}{f}}$ & $\approx$ & $\begin{array}{l}\stackrel{R}{\infty} \\
\stackrel{\infty}{\simeq} \\
=\end{array}$ & $\stackrel{\infty}{2}$ & $\stackrel{\sharp}{\longrightarrow}$ & 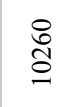 \\
\hline & & - & $\sim$ & $m$ & 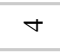 & in & 0 & $r$ & $\infty$ & $a$ & $\stackrel{\circ}{\circ}$ & $=$ \\
\hline
\end{tabular}


Table 3. Carpenter's Likelihood Ratios

\begin{tabular}{|lcc|}
\hline Historical features & LR+ & LR- \\
Worst headache & 1.25 & 0.24 \\
Thunderclap & 1.34 & 0.74 \\
Exertion at onset & 1.70 & 0.88 \\
Onset during intercourse & 1.20 & 1.00 \\
Loss of consciousness & 1.87 & 0.91 \\
Blurred vision & 3.14 & 0.85 \\
Nausea & 1.15 & 0.74 \\
\hline
\end{tabular}

\begin{tabular}{|lll|} 
Examination features & LR+ & LR- \\
Meningism & 6.59 & 0.78 \\
Vomiting & 1.92 & 0.52 \\
Unable to walk & 2.36 & 0.85 \\
Altered mental status & 2.18 & 0.87 \\
Focal neuro deficit & 3.26 & 0.81 \\
\hline
\end{tabular}

This article is protected by copyright. All rights reserved. 
Utility of lumbar puncture after a normal brain CT scan in patients presenting to the emergency department with suspected Subarachnoid Haemorrhage, a new more rational approach?

Pouryahya P (1,2,4), Haydon R (3,4), Meyer A (1,2,4), Easaw-Mamutil N (4), Zhi Shawn Tan Y (4), Teng Hui Wen G (4).

1) Casey hospital, Emergency Department, Program of Emergency Medicine, Monash Health, Victoria

2) Monash Emergency Research Collaborative, School of Clinical Sciences, Monash Health, Monash University, Victoria

3) Monash Medical Centre, Emergency Department, Program of Emergency Medicine, Monash Health, Victoria

4) Faculty of Medicine, Nursing and Health Sciences, Monash University, Victoria

Corresponding author:

Dr. Pourya Pouryahya

Casey hospital, 62 Kangan drive, Berwick, Victoria 3806, Australia

email: Pourya.Pouryahya@monashhealth.org

Pourya.Pouryahya@monash.edu

This article is protected by copyright. All rights reserved. 


\section{University Library}

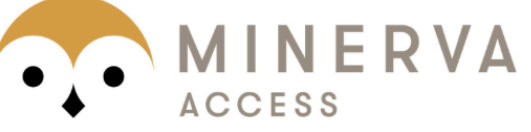

A gateway to Melbourne's research publications

Minerva Access is the Institutional Repository of The University of Melbourne

Author/s:

Pouryahya, P;Haydon, R;Meyer, A;Easaw-Mamutil, N;Tan, SYZ;Teng, GHW

Title:

Utility of lumbar puncture after a normal brain computed tomography scan in patients presenting to the emergency department with suspected subarachnoid haemorrhage: A new more rational approach?

Date:

2020-03-20

Citation:

Pouryahya, P., Haydon, R., Meyer, A., Easaw-Mamutil, N., Tan, S. Y. Z. \& Teng, G. H. W. (2020). Utility of lumbar puncture after a normal brain computed tomography scan in patients presenting to the emergency department with suspected subarachnoid haemorrhage: A new more rational approach?. EMERGENCY MEDICINE AUSTRALASIA, 32 (5), pp.756-762. https://doi.org/10.1111/1742-6723.13502.

Persistent Link:

http://hdl.handle.net/11343/275557 